\title{
State Estimation of Electric Power Systems Including FACTS Models (SVC and STATCOM)
}

\author{
Irina Kolosok ${ }^{1}$, Alexander Tikhonov ${ }^{2}$, Anatolijs Mahnitko ${ }^{3 *}$ \\ ${ }^{1-2}$ Energy System Institute SB RAS, ${ }^{3}$ Riga Technical University
}

\begin{abstract}
Nowadays, FACTS devices are among the most promising electrical network technologies. The typical representatives of parallel FACTS devices are a static synchronous compensator (STATCOM) and a state var compensator (SVC). The paper deals with the issues of modeling SVC and STATCOM when solving the problem of power system state estimation. The algorithms developed by the authors for embedding the SVC and STATCOM models in the state estimation problem are presented. The paper considers the results of calculations using the algorithms devised for a section of the $500 \mathrm{kV}$ network of the Irkutsk electric power system. A comparative analysis of the obtained results is made.
\end{abstract}

Keywords - FACTS, state estimation, state var compensator, static synchronous compensator.

\section{INTRODUCTION}

Successful (reliable, quality and economical) operation of Smart Grid requires a wide range of advanced technical instruments and technologies that afford the opportunity to endow the network with active-adaptive qualities.

In the international and national practices, there is a persistent trend toward adopting the power electronics controlled devices in the electrical networks of power systems, i.e., Flexible Alternating Current Transmission Systems (FACTS). Today, FACTS are among the most promising electrical network technologies [1]-[3], which make it possible for the electrical network to turn from a passive facility for electricity transportation into the facility which takes an active part in control of the network operation. This technology provides control of interrelated parameters, including impedances, currents, voltages, phase angles, oscillation damping at different frequencies, etc., and opens up new opportunities to control electric power systems.

The FACTS devices can be used to increase the transfer capability, improve static and dynamic stability, and provide better power quality.

Currently, there are intensive studies on the use of models of such devices in the equivalence circuits for steady state calculation both in Russia [2], [4], [5] and in other countries [3], [6], [7].
To form the calculation model of the current state of the electric power system, we use the state estimation methods. State estimation is the most important procedure that provides reliable and quality data to control electric power systems [8], [9]. The result of the state estimation is an electric power system steady state (current state) calculated on the basis of state variable measurements and the data on the network topology. The model of power system current state obtained from the state estimation is used for solving the problem of operation and emergency control. The development of mathematical models for power system state estimation considering FACTS as well as the research into the impact of these models on the efficiency of state estimation methods is a relevant problem today. In recent years, such research is actively performed in other countries [10]-[12]. In Russia, this research is at initial stage [13].

This paper deals with the FACTS devices, which when installed at the facilities of power systems afford the possibility of controlling stability and compensating for voltage oscillations in the network, caused by frequent changes in the consumed reactive power. As a result, the efficiency is enhanced, the load of power plants is reduced, and the service life of the equipment is extended. These devices include static synchronous compensators (STATCOM) and static var compensators (SVC).

The paper presents modified state estimation algorithms for the case when STATCOMs and SVCs are included in the equivalence circuit of an electric power system. To check the efficiency of the algorithm, the comparative calculations were performed for a $500 \mathrm{kV}$ network section of the Irkutsk power system with either STATOMs or SVCs installed at the nodes of the scheme.

\section{MODELING OF FACTS DEVICES (SVC, STATCOM) FOR STEADY STATE CALCULATION}

One of the FACTS devices is static var compensator (SVC). This is a multifunctional static device providing stable voltage and gradual or stage-by-stage changes in the reactive power consumed and (or) supplied by it at the buses of its connection. The static var compensator is based on the storage elements (capacitances, inductances), thyristor switched capacitors (TSC), and thyristor controlled reactors (TCR).

\footnotetext{
* Corresponding author.

E-mail address: mahno@eef.rtu.lv
} 


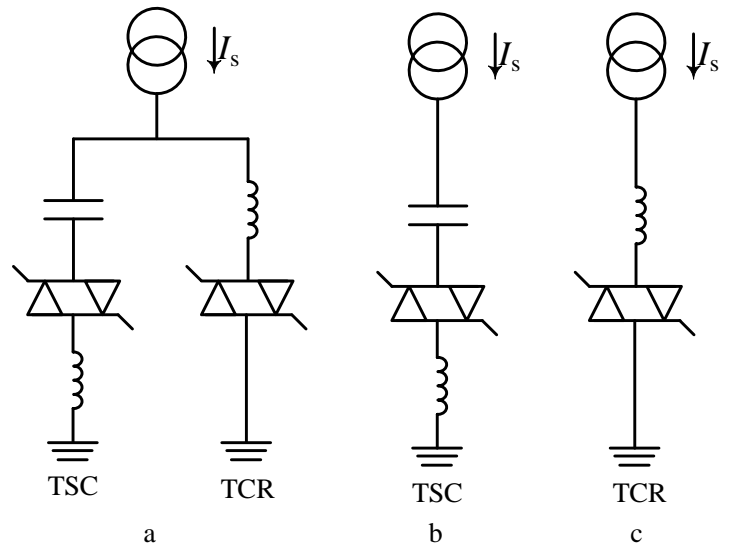

Fig. 1. A design of SVC: a) TSC+TCR; b) TSC; c) TCR.

In most cases, the static compensator consists of a TSC and a TCR (Fig. 1a). There can also be other combinations of the devices, for example, a separately designed TSC (Fig. 1b) or TCR (Fig. 1a). According to [3], SVC can consume or generate reactive power in order to control several specified parameters (normally voltage at some point of the network).

The working characteristic of SVC is presented in Fig. 2.

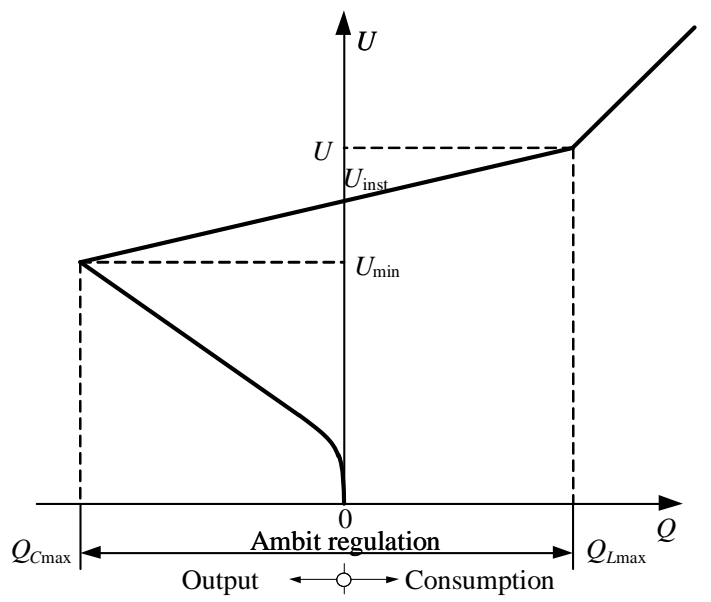

Fig. 2. Working characteristic of SVC.

Within the control range from $Q_{C \max }$ to $Q_{L \max }$, the characteristic has a slope $(2 \%$ to $5 \%)$ determined by the frequency droop. Beyond the control range, the characteristic changes linearly depending on the values of voltage $U$ and capacitive $X_{C}$ and inductive $X_{L}$ reactances of SVC. Gradual control of reactive power in SVC is carried out by changing the firing angle of the thyristors in the reactor. In order to specify the admittance and maintain the specified voltage at node, it is necessary to determine the angle $\alpha$.

When solving the state estimation problem and calculating steady state, the reactive power of the shunt at a node $i$ is determined by the equation:

$$
Q_{i}^{\mathrm{H}}=U_{i}^{2} b_{i \mathrm{H}}
$$

and is used in the equation of nodal balance for reactive power:

$$
Q_{i}=Q_{i \mathrm{G}}+Q_{i \mathrm{H}}+\sum_{j \in \omega_{i}} Q_{i j}+Q_{i}^{\mathrm{H}}
$$

where: $U_{i}$ - voltage at node $i ; b_{i \mathrm{H}}$ - the nodal shunt admittance; $Q_{i \mathrm{G}}, Q_{i \mathrm{H}}$ - reactive power generated and consumed at node $i$; $\sum_{j \in \omega_{i}} Q_{i j}$ a sum of reactive power flows along the lines incident to node $i ; \omega_{i}-$ a set of nodes incident to the $i$-th one.

The authors of [3], [4], and [7] show that the SVC admittance, depending on angle $\alpha$, is determined by the expression:

$$
b_{\mathrm{SVC}}(\alpha)=\frac{1}{X_{C} X_{L}}\left\{X_{L}-\frac{X_{C}}{\pi}[2(\pi-\alpha)+\sin 2 \alpha]\right\} \text {. }
$$

By substituting (3) to (1), we obtain an expression for the calculation of SVC reactive power:

$$
Q_{i}^{\mathrm{SVC}}=-\frac{U_{i}^{2}}{X_{C} X_{L}}\left\{X_{L}-\frac{X_{C}}{\pi}[2(\pi-\alpha)+\sin 2 \alpha]\right\} \text {. }
$$

The authors of [3] and [4] considered the SVC model, which in an explicit form includes the control angle $\alpha$. In these studies, the expression for the SVC reactive power is represented as (4) (in our notations).

Linearized equations for SVC in the steady-state calculation in the $k$-th iteration are written as follows:

$$
\left[\begin{array}{c}
\Delta P_{i} \\
\Delta Q_{i}
\end{array}\right]^{(k)}=\left[\begin{array}{cc}
0 & 0 \\
0 & \frac{2 U_{i}^{2}}{\pi X_{L}}[\cos 2 \alpha-1]
\end{array}\right]^{(k)}\left[\begin{array}{c}
\Delta \delta_{i} \\
\Delta \alpha
\end{array}\right]^{(k)}
$$

The same as most of the FACTS devices, STATCOM represents a thyristor-controlled reactive power source that maintains a specified voltage value by consuming or generating reactive power at the connection point without using additional external reactors or high-power capacitor banks.

STATCOMs involve either gate turnoff thyristors (GTOT) or integrated gate-commutated thyristors (IGCT), or insulatedgate bipolar transistors (IGBT).

STATCOM can include either a voltage converter or a current converter. Fig. 3 presents a simplified scheme of STAT$\mathrm{COM}$ with a voltage converter.

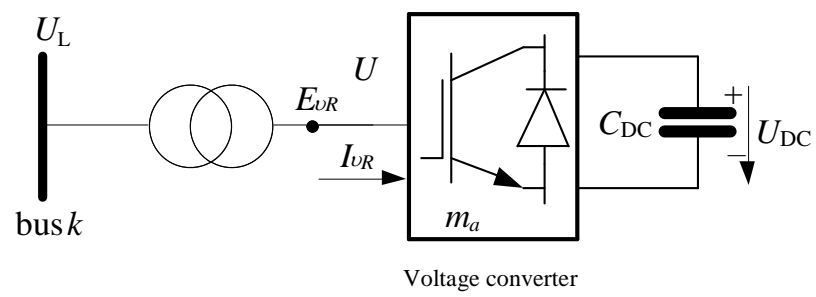

Fig. 3. STATCOM design with a voltage converter. 
The reactive power flow $Q$ between the voltage converter and the AC electric power system is controlled by changing the value of the output voltage of the voltage converter, $U$. When the value $U$ exceeds the value of voltage in line, $U_{\mathrm{L}}$, the STATCOM operates in a capacitive mode and reactive power is generated. As the value $U$ declines below the value of voltage in line $U_{\mathrm{L}}$, STATCOM operates in an inductive mode. In this case, the reactive power is consumed. When the voltages are equal $U_{\mathrm{L}}=U, Q=0$.

The parameters specified in STATCOM include the voltage setting $U_{\text {ref, voltage droop }} X_{\mathrm{ST}}$, and ranges of change in the current $I_{\min }, I_{\max }$. Figure 4 demonstrates the STATCOM volt-ampere characteristics.

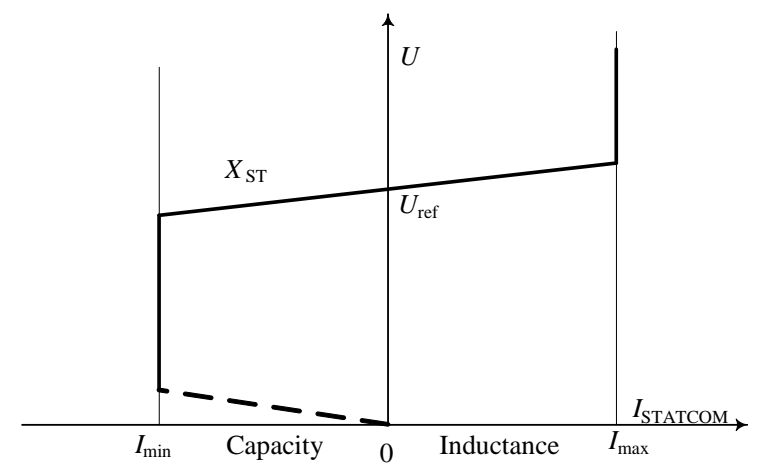

Fig. 4. STATCOM volt-ampere characteristics.

STATCOM devices use gate-turnoff and insulated-gate bipolar technologies with pulse-duration modulation, which makes it possible to regulate the amplitude and phase of voltage thanks to fast switchings of GTO or IGBT elements. High modulation frequencies allow a considerable reduction in the level of harmonics in the output signals.

Thyristor converter of STATCOM provides an exchange of reactive power between the network phases. Thus, STATCOM can both generate and consume reactive power.

STATCOM is characterized by fast operation and small size. It can regulate both the value and the phase of voltage in the electrical network it is connected to. In the case the DC section has a storage device, STATCOM can also regulate active power.

STATCOM can be represented as a voltage source connected to the network through shunt $Z_{v R}$ (Fig. 5) whose admittance p.u. equals droop.

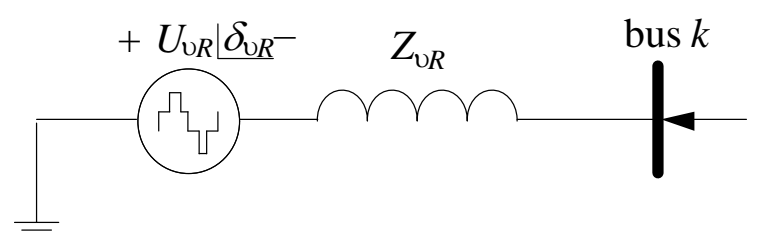

Fig. 5. Equivalence scheme of STATCOM design.

In this case, the controlled variables will be represented by voltage amplitude $U_{v R}$ and angle $\delta_{v R}$ of the voltage converter, which are state variables.
For active and reactive power components of the converter and bus $k$, we can write the following set of equations [3]:

$$
\begin{aligned}
& P_{v R}=U_{v R}^{2} G_{v R}+U_{v R} U_{k}\left[G_{v R} \cos \left(\delta_{v R}-\theta_{k}\right)+B_{v R} \sin \left(\delta_{v R}-\theta_{k}\right)\right] \\
& Q_{v R}=-U_{v R}^{2} B_{v R}+U_{v R} U_{k}\left[G_{v R} \sin \left(\delta_{v R}-\theta_{k}\right)-B_{v R} \cos \left(\delta_{v R}-\theta_{k}\right)\right] \\
& P_{k}=U_{k}^{2} G_{v R}+U_{k} U_{v R}\left[G_{v R} \cos \left(\theta_{k}-\delta_{v R}\right)+B_{v R} \sin \left(\theta_{k}-\delta_{v R}\right)\right] \\
& Q_{k}=-U_{k}^{2} B_{v R}+U_{k} U_{v R}\left[G_{v R} \sin \left(\theta_{k}-\delta_{v R}\right)-B_{v R} \cos \left(\theta_{k}-\delta_{v R}\right)\right]
\end{aligned}
$$

The fully written linearized model (6) is presented in [3].

Let us consider how the proposed approaches to the power system state estimation problem can be implemented.

\section{The Problem of Power System State Estimation}

The state estimation problem of an electric power system implies the calculation of state variables, which is based on the data of measurements [8], [14]. The measurements applied in the state estimation are mainly represented by remote measurements received from SCADA systems: magnitudes of nodal voltage $U_{i}$, generation of the active $P_{\mathrm{G}}$ and reactive $Q_{i j}$ power at nodes, power flows in the transformers and lines $P_{i j}, Q_{i j}$, less often - currents at nodes and in lines $I_{i}, I_{i j}$. Accordingly, the vector of measurements looks like:

$$
\bar{y}=\left\{P_{i}, Q_{i}, P_{i j}, Q_{i j}, U_{i}, I_{i}, I_{i j}\right\} .
$$

When solving the state estimation problem, we introduce a notion of the state vector $x$ with dimension $2 n-1$ (where $n$ is the number of nodes of the calculation scheme). The state vector includes the voltage magnitudes $U$ and the phase angles $\delta x=(\delta, U)$, except for the fixed phase of base node. Such a vector of state uniquely determines all the other state variables.

Mathematical statement of the state estimation problem is reduced to the minimization of the objective function

$$
J(x)=(\bar{y}-y(x))^{\mathrm{T}} R_{y}^{-1}(\bar{y}-y(x))
$$

i.e., to the search of the estimates of the state vector $\hat{x}$; here $R_{y}{ }^{-1}$ is a diagonal matrix of the weighting coefficients whose elements are inverse to the variances of the measurements that characterize their accuracy.

Due to the nonlinear dependence of $y(x)$, the problem is solved iteratively. In each iteration, a normalized system of equations is solved with respect to the correction vector:

$$
\Delta x_{k}=\left[H_{k}^{T} R_{y}^{-1} H_{k}\right]^{-1} H_{k}^{T} R_{y}^{-1}\left[\bar{y}-y\left(x_{k}\right)\right]
$$

where $H_{k}=\frac{\partial y}{\partial x}$ is the matrix of Jacobi, calculated in the $k$-th iteration. 


\section{MOdeling OF SVC AND STATCOM IN STATE EstimA- TION OF ELECTRIC POWER SYSTEM}

When modeling SVC, the angle $\alpha$ is specified as a state vector component and is determined directly in the process of solving the state estimation problem.

In the calculation of corrections to the state vector $x$ by (9), the derivative of the injection measurement $Q_{i}$ with respect to voltage $U_{i}$ is taken equal to:

$$
\frac{\partial Q_{i}}{\partial U_{i}}=\sum_{j \in \omega_{i}} \frac{\partial Q_{i j}}{\partial U_{i}}+2 U_{i} b_{i \mathrm{H}}
$$

When using the $S V C$ model with the control angle $\alpha$ in the state estimation problem, $S V C$ is modeled by the variable of susceptance at node $i$ at which the $S V C$ is installed, and $\alpha$ is included in the state vector $x$ instead of $U_{i}$, which is fixed. A derivative of the injection measurement at the node with $S V C$ with respect to $\alpha_{i}$ will be equal to:

$\frac{\partial Q_{i}}{\partial \alpha_{i}}=-\frac{U_{i}^{2} b_{L}}{\pi}(-2+2 \cos 2 \alpha)=\frac{2 U_{i}^{2} b_{L}}{\pi}(1-\cos 2 \alpha)$.

An algorithm for the calculation

1. Specify the admittance $b_{\mathrm{SVC}}$ at a necessary node $i$, using reference data $b_{\mathrm{SVC}}=\frac{Q_{\mathrm{SVC}}}{U_{\mathrm{SVC}}^{2}}$.

2. Fix voltage at node $i$ at a required value, by specifying it by the measurements with a zero (a very small) variance. The state vector $x$ will include $\alpha_{i}$ for this node

3. Specify the initial approximations of the vector $x=x_{0}$, at which specify $\alpha_{i 0}=\pi / 2$ for the node where the SVC is installed; $U_{i 0}=U_{i n o m}$; for the other nodes: $\delta_{i 0}=0$; $U_{i 0}=U_{\text {inom. }}$

4. Calculate the corrections in iterations by the method of Newton, using (9). In this case, there will be one nonzero element calculated by ( 9 ) in the column of derivatives $\frac{\partial Q_{i}}{\partial \alpha_{i}}$ in the matrix $\frac{\partial y}{\partial x}$. At node $i$, it is necessary to specify the measurement of the nodal injection $Q_{i}$.

STATCOM is modeled as a voltage source connected to the network through shunt $Z_{v R}$. The control parameters of STATCOM (voltage $U_{v R}$ and angle $\delta_{v R}$ of a voltage con- verter) represent the state vector components in state estimation. Therefore, such a model is easily included in the state estimation algorithm.

1. We will dwell on the calculation algorithm in more detail.

2. At node $i$, where voltage should be maintained constant, we specify a branch with a fictitious node.

3. Branch parameters: resistance $R=0 \Omega$, the value of reactance $X$ is assumed equal to a droop of the STATCOM characteristic presented in Fig. 4. In our case study, $X=0.086 \Omega$.

4. Fix voltage at node $i$ at a required value by specifying it with the measurement with a zero (very small) variance. Node $i$ is assumed to be a transit one.

5. Fictitious node is specified by a node transit with respect to active power.

6. Make state estimation. In the process of calculation, we obtain the reactive power injection $Q_{\text {STATCom }}$ at the STATCOM connection point, which is necessary to maintain the required voltage value.

7. Check the condition: $Q_{\min }<Q_{\text {StATCOM }}<Q_{\max }$, into which, as is shown in [5], the condition $I_{\min }<I_{\text {STATCOM }}<$ $I_{\max }$ can be transformed within the control range.

The condition $U_{\min }<U_{\text {STATCOM }}<U_{\max }$ is controlled when solving the problem of state estimation.

7.1. In the case condition (9) is met, we go to p. 6.

7.2. In the case condition (9) is not met, the algorithm of considering the inequality-constraints on unmeasured variables, which is implemented in the software "Otsenka", operates [14].

8. Exit.

\section{Calculation Results}

A scheme for testing the suggested methods is represented by a 19-node scheme of the Irkutsk electric power system presented in Fig. 6.

For modeling, we used STATCOM with two inverter units, $50 \mathrm{MVar}$ each, at voltage of $11 \mathrm{kV}$, and SVC with two inverter units, $40 \mathrm{MVar}$ each, at voltage of $11 \mathrm{kV}$.

STATCOM and SVC are modeled at a low side of autotransformer of the "Irkutskaya" substation (nodes 17, 18, and 19). When modeling STATCOM, the scheme was supplemented with fictitious nodes 177, 188, 199 and branches 17177, 18-188, 19-199.

The results of comparative calculations for STATCOM and SVC are presented in Table I. 


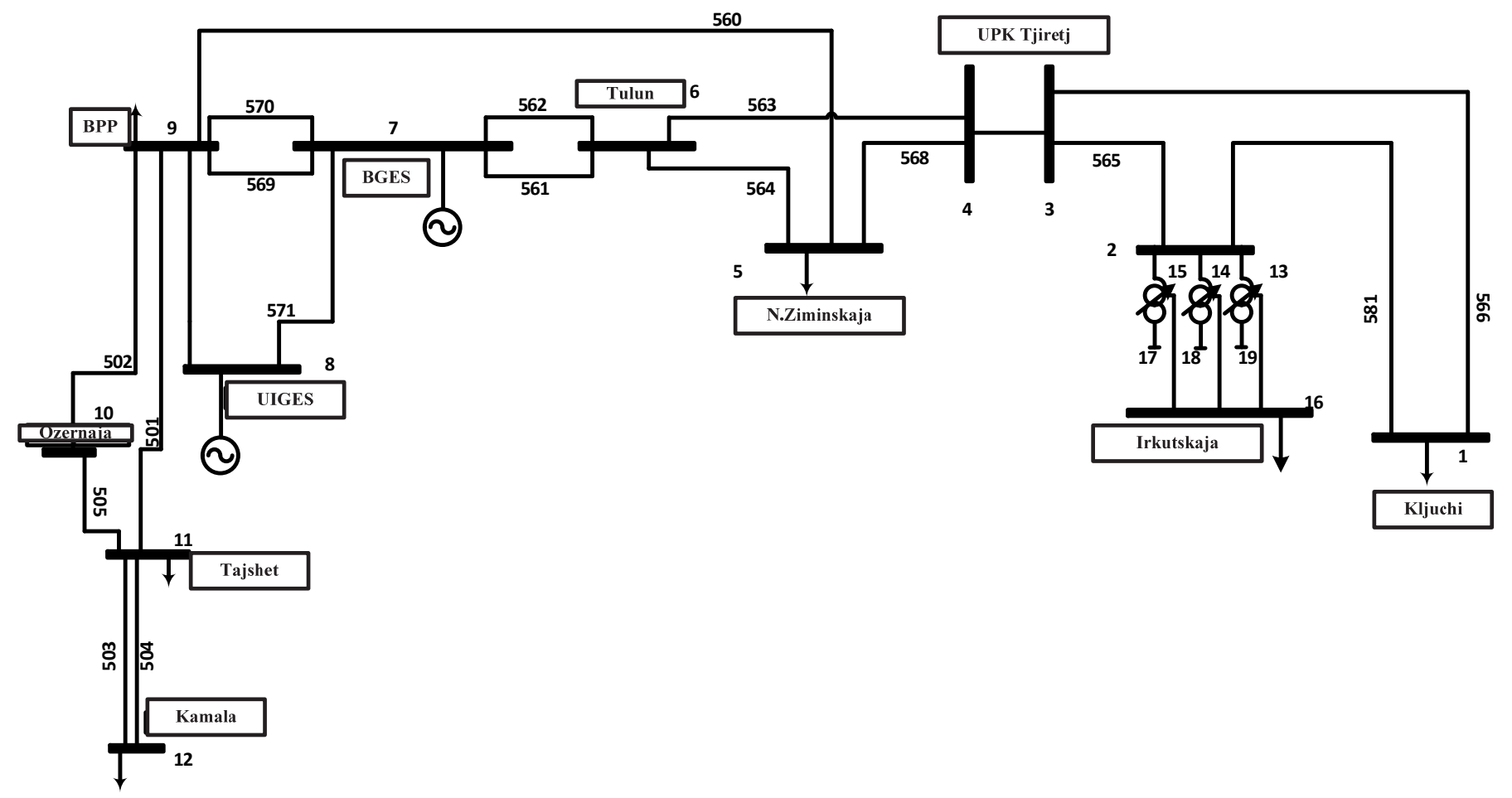

Fig. 6. A scheme of the Irkutsk electric power system.

TABLE I

CALCULATION RESUlts

\begin{tabular}{|c|c|c|c|c|c|c|c|c|c|}
\hline Node & $U_{\text {meas }}$ & $U_{\mathrm{se}}$ & $\delta$ & $P_{\text {meas }}$ & $P_{\mathrm{se}}$ & $Q_{\text {meas }}$ & $Q_{\mathrm{se}}$ & $b_{\mathrm{SVC}}$ & $\alpha$ \\
\hline No. & $\mathrm{kV}$ & $\mathrm{kV}$ & degree & MW & MW & MVar & MVar & mcSim & \\
\hline \multicolumn{10}{|c|}{ Calculation with STATCOM } \\
\hline 16 & 220 & 224 & -7.9 & -381 & -382 & -402 & -402 & & \\
\hline 17 & 10.5 & 10.5 & -8.0 & - & 0.0 & & & & \\
\hline 18 & 10.5 & 10.5 & -8.0 & - & 0.00 & & & & \\
\hline 19 & 10.5 & 10.5 & -8.0 & - & 0.00 & & & & \\
\hline 177 & - & 11.4 & -8.0 & - & 0.00 & & 11 & & \\
\hline 188 & - & 11.4 & -8.0 & - & 0.00 & & 11 & & \\
\hline 199 & - & 11.4 & -8.0 & - & 0.00 & & 11 & & \\
\hline \multicolumn{10}{|c|}{ Calculation with SVC } \\
\hline 16 & 220 & 223.64 & -7.9 & -382 & -394 & -402 & -396.67 & & \\
\hline 17 & 10.5 & 10.55 & -8.0 & - & 0.00 & & 10 & 0.090 & -0.934 \\
\hline 18 & 10.5 & 10.55 & -8.0 & - & 0.00 & & 10 & 0.090 & -0.934 \\
\hline 19 & 10.5 & 10.55 & -8.0 & - & 0.00 & & 10 & 0.090 & -0.934 \\
\hline
\end{tabular}


The data of Table I demonstrate the calculation results for the cases of connecting two different reactive power control devices (SVC and STATCOM) that virtually coincide (within a specified accuracy of calculation).

The analysis of the algorithms shows that for modeling SVC, it is necessary to include additional components in the vector of state, but this makes it possible to determine the angle of control $\alpha$ in real time [13]. The algorithm allows us to form a mathematical model of an energy system with embedded SVC model, which does not require additional preparatory or subsequent calculations and can be used for off-line operation in various software packages. The main flaw of the algorithm is the fact that it is hard to implement and should be adjusted to a specific program of state estimation, considering its specific features and constraints of the applied methods.

STATCOM is modeled by an additional branch with a voltage source at the node where STATCOM is installed. The algorithm is easily implemented with the minimum labor efforts and can be implemented virtually in any state estimation software (Cosmos, ANARES, Otsenka, etc.) without changes in programming code and introduction of new components in the vector of state as in the case of including the SVC model in the power system state estimation problem. This algorithm does not require direct participation of an operator in the calculations and can operate in real time. The necessary condition for the implementation of the STATOM model in the state estimation software is availability of a module for considering inequality-constraints on measured and unmeasured variables. It should also be noted that compared to SVC STATCOM can be applied when voltage declines greatly. Moreover, the STATCOM response to changes in operating conditions is faster and the device does not generate harmonic oscillations.

\section{CONCLUSION}

The paper shows the relevance of including the models of FACTS devices in the equivalent circuit when solving the power system state estimation problem. Consideration is given to the models of SVC and STATCOM, which are used in the steady state calculation. The modified state estimation algorithms developed for modeling the SVC and STATCOM devices provide voltage stabilization and gradual or stage-bystage change in the consumed and (or) supplied reactive power. The calculations made for a fragment of the $500 \mathrm{kV}$ Irkutsk power system demonstrated high-speed operation and good convergence of the developed algorithms, which makes it possible to use them for obtaining the estimates in real time.

\section{ACKNOWLEDGMENT}

The research was supported by the grant 4711.2014 .8 of Leading Scientific School of the Russian Federation.

\section{REFERENCES}

[1] V. E. Fortov and A. A. Makarov, A concept of Russia's intelligent power system with active-adaptive network, Moscow: JSC "NTC FSC EES", 2012, p. 235.

[2] E. V. Ametistov, Fundamentals of modern energy. Modern electric power engineering, Moscow: Publishing MEI, 2010, V.2, p. 632.

[3] E. Acha, C. R. Fuerte-Esquivel, H. Ambriz-Perez, C. Angeles-Camacho, FACTS. Modeling and Simulation in Power Networks, England: JohnWilley\&Sons, Ltd, 2004, p. 403.

[4] N. I. Voropai, O. B. Osak, "Development of equipment and control systems of large-scale energy systems," Scientific and technical report, code 2008-0-2.7-31-01-007, Irkutsk, 2009.

[5] P. M. Erokhin, V. G. Neuimin, A. S. Alexandrov and D. M. Maksimenk, "Modeling of FACTS devices in the problems of calculation and optimization of energy system operation," Bulletin of NTC EES, No. 66 (1). pp. 22-28, 2012.

[6] Y. H. Song and A. T. Johns (eds.), Flexible AC Transmission Systems (FACTS), London: Institution of Electric Engineers, 1999.

[7] N. G. Hingorani and L. Gyugyi, Understanding FACTS: concepts and technology of flexible AC transmission systems, Wiley-IEEE Press. 2000, pp. 431-432.

[8] A. Monticelly, "Electric power system state estimation," in Proc. of the IEEE, vol. 88, issue 2, Feb. 2000. pp. 229-240. http://dx.doi.org/10.1109/5.824004

[9] A. Z. Gamm and I. N. Kolosok, "Test Equations and Their Use for State Estimation of Electrical Power System," Power and Electrical Engineering: Scientific J. of Riga Technical University. Riga: RTU Press, pp. 99$105,2002$.

[10] T. Okon and K. Wilkosz, "WLS State Estimation in Polar and Rectangular Coordinate Systems for Power System with UPFC: Significance of Types of Measurements," in Proc. of the Int. Conf.-PowerTech, Trondheim, Norway, June 19-23, 2011.

[11] A. Zamora-Cardenas and C. R. Fuerte-Esquivel, "State estimation of power systems containing facts controllers," Electric Power Systems Research, vol. 81, pp. 995-1002, 2011. http://dx.doi.org/10.1016/j.epsr.2010.12.009

[12] B. Xu and A. Abur, "State Estimation of Systems with Embedded FACTS Devices," in Proc. of the Int. Conf., PowerTech'2013, Bologna, Italy, June 23-26, 2003. http://dx.doi.org/10.1109/PTC.2003.1304122

[13] A. V. Tikhonov, "Modeling of static var compensators in the state estimation problem of electric power system," System studies in energy 2014. The papers of young scientists of ESI SB RAS, Issue 44, pp. 64-72.

[14] A. Z. Gamm and I. N. Kolosok, Bad data detection in measurements in electric power systems, Novosibirsk: Nauka, 2000, p. 152. 